\title{
The Use of Metapatterns for Research into Complex Systems of Teaching, Learning, and Schooling
}

\section{Part I: Metapatterns in Nature and Culture}

\author{
TYLER VOLK \\ New York University (USA) \\ JEFFREY W. BLOOM \\ Northern Arizona University (USA)
}

\begin{abstract}
We justify the concept of metapatterns as functional patterns or functional principles that are common to a large set of systems that encompass both biology and culture, by starting with the fact that evolved systems, whether biological or cultural, are produced from any iterative sequence of replication, variation, and selection. Therefore the systems that result, with specific functional parts, are formed as wholes that fit particular contexts. The principle of convergence in biological evolution, in which similar structures are independently evolved, is the model that can be extended even beyond biology. If the contexts of evolved systems across widely separated scales are similar, the resulting evolved systems can exhibit convergences that themselves occur at diverse scales. These grand convergences are the metapatterns. For example, the functional advantage of dynamically separating systems from their environments sets the context for the evolution of the metapattern of borders across various scales. We outline fifteen additional examples of metapatterns. We also examine the correspondences and differences between metapatterns as a multi-scale approach to systems and the approach from complexity science. We suggest that metapatterns could serve as tools for thinking about a diverse range of topics, and could thereby motivate the transference of generalizations. Finally, we propose that because metapatterns are employed in human thought, they will be useful in formulating new questions for education research, which is the subject of the companion paper.
\end{abstract}




\section{Introduction}

In 1979, the anthropologist and epistemologist Gregory Bateson coined the term metapattern to refer to a pattern of patterns (Bateson, 1979). Bateson pointed out that it is by analyzing patterns - the interrelated structural, functional, and dynamical aspects of systems, their parts, and their contexts- that we draw connections and ultimately make explanatory generalizations. Indeed, everything from atoms to the story of Adam and Eve has pattern, a shape, a structure, an arrangement of parts into a specific whole. While we normally think of patterns as spatial or geometric aspects of systems, we emphasize that the word pattern can fruitfully be applied as well to the relations among parts within systems, for instance in the description of functional relationships, and also to the temporal dynamics of systems as patterns in time.

Bateson's own example of an analysis using metapatterns began with a crab. The crab's two front limbs, though one is enormous and much more threatening compared to the other, share a common pattern in their arrangement of parts and joints. The crab's appendages can then be matched against a lobster's, and then a horse's limbs put side to side along those of a human, thus widening the circles of comparison. But Bateson did not note that the mammal's limb pattern can be judged against that of the arthropod's, and they seem to differ in huge ways. Nonetheless, all these limbs do have joints and, even more generally, are linear structures that function, in part, as columns to hold up the bodies of these organisms away from the ground or marine sediments, as well as to transfer forces during movement, thereby creating a relationship between the creatures and their solid substrates.

Another point that Bateson did not explicitly address was the level of generality to which the term metapattern does or does not apply. He clearly was aware that such analysis could extend to very high levels, such as mental processes. In his chapter, "Multiple Versions of Relationship," he compared the advantageous genetic mixing spawned by two sexes (a biological, gene-level binary), to depth perception gained from binocular vision (a physiological binary), and then to Macbeth's conscious understanding that he is hallucinating when he sees but does not feel the dagger before him (a psychological binary). In this paper, we will be explicit about the high, general levels of analysis required for metapatterns. We will use the term metapattern to refer to common patterns that occur across scales that include biological systems, cultural systems, and even mental systems (Volk, 1995). Although patterns are also crucial to the understanding of systems not subject to evolutionary design, such as those of physics, chemistry, and geology-indeed many examples of metapatterns in our list also occur in such realms-we put these purely physical-chemical systems to the side 
for the present discussion, because one cannot invoke functional explanations for them, a concept already alluded to as crucial for understanding the existence of metapatterns.

To continue with our expansion from Bateson's example, consider that the linear legs of crabs and people (both animals) share a design with trees, whose trunks and limbs act as columns that hold the leaves up toward the sun and away from the ground. And human engineers, from aboriginal peoples to the builders of the Eiffel Tower, have used columns to transmit structural forces. The study of universal engineering principles to draw connections between shapes created by nature and humans is one important route to finding metapatterns. We, however, offer the idea that an approach to education and research based on metapatterns can go even further. In addition to their existence as engineering design principles, metapatterns apply to the more psychological realms, which is why metapatterns can be useful in educational research (see Part II). The metapattern of the tube (in other words, linear shapes) includes instances in which structural columns or conduits have been employed as metaphors, from human mythology to conceptual modeling.

A corn stalk, for example, is key to a sand painting from the Navajo Blessing Way ceremony (Bahti, 2000), believed to be the oldest of all Navajo ceremonies and which has been called, metaphorically, a ceremonial backbone or spinal column, because a song from the Blessing Way concludes other ceremonial "Ways." In the sand painting, the corn stalk is depicted as a bridge from the underworld upwards, a tube between earth and sky, a pattern that we note is "in line" with the universal iconography of the Axis Mundi, or world axis, as elucidated by scholars of mythology such as Joseph Campbell (1974) and Mircea Eliade (1972). Lines connecting zones or bubbles in the abstract spaces created by various modeling activities, which use tubes in the metaphoric sense of relationship, are virtually ubiquitous in creating associative semantic webs and graphic organizers, such as clustering (Rico, 1976, 1983/2000), concept maps (Novak and Gowin, 1984) and context maps (Bloom, 1995).

In the corn stalk of Navajo iconography and in the relational lines of concept maps and semantic webs, the tube metapattern appears as a visual metaphor. Aquestion about the nature of the relationship between metapatterns and metaphors was raised by one of our reviewers. It is apparent that some instances of a metapattern do occur as metaphors, in which concrete imagery is used to represent things that are not physical. Following the reasoning of Lakoff and Johnson (2003), the occurrences of physically-based metaphors to represent abstractions are the very means by which the mind conceives of many (if not all) more abstract principles and concepts. In contrast, when we look at tree trunks and crab appendages, the metapattern is 
truly there as a support column. So although metapatterns have important metaphoric instances they are not only metaphors. The fact that metapatterns appear in metaphoric constructions shows that the metapatterns are functional not only in the physical structures made by biology and culture but also in the shapes of imagination and reasoning.

Having provided an example of a metapattern (the tube) and a sense of its range-biological, cultural, and mental-we will next justify why metapatterns are to be expected, to alleviate any qualms that we are making up the fact of their existence. Indeed, the occurrences of each metapattern are so widespread that the terms transphenomenal and transdisciplinary (Davis and Phelps, 2005) can be applied, because metapatterns bridge not only diverse phenomena but diverse disciplines. We believe that a set of metapatterns, as we outline below, could serve as a basis for a visual typology of functional patterns and processes. Furthermore, because each metapattern is fairly simple as a functional principle or small set of related principles, it might turn out to be the case that the most compelling reason for figuring out metapatterns is to help foster creative approaches to learning, including enhanced self-learning and education research. But first, we will discuss their rationale.

\section{Metapatterns as Functional, Evolved Convergences}

Volk (1995) called metapatterns "functional attractors." However, neither Volk nor Bloom (2005a,b) formally developed the reasoning behind the existence of metapatterns. And neither did Bateson. Why should wide-spanning dynamical patterns come into being? As one reviewer asked, perhaps there are only a few ways of accomplishing things? A formal argument for expecting metapatterns is a necessary part to any application of the metapatterns in education, say in curriculum design. Furthermore, for using the metapatterns in educational research we want to know why we would expect them to occur at different scales in social structures, such as in the classroom itself or in the dynamics of children's discussions in active learning, for example about arguments around the meaning of density (see Part II).

A key point to our providing a rationale for the existence of metapatterns is the concept of convergence. The use of this term is well established in evolutionary biology, which we review first before extending it substantially to include culture.

In biology, the idea behind convergence is that natural selection can produce similar forms in similar environmental contexts. For example, the independently evolved wings of birds, bats, and butterflies are all flat because they share a design requirement for high contact area with air. Another celebrated example comes from American cacti and African succulents, 
which separately evolved from only more distantly related leafy ancestors. Cacti and succulents both have strikingly similar water-storing, bulbous stem and leaf parts as adaptations for life in a desiccated environment, and botanical gardens often display paired examples from both groups alongside each other as biological mirrors, as reflected in their spines and geometric shapes (see Figure 1).
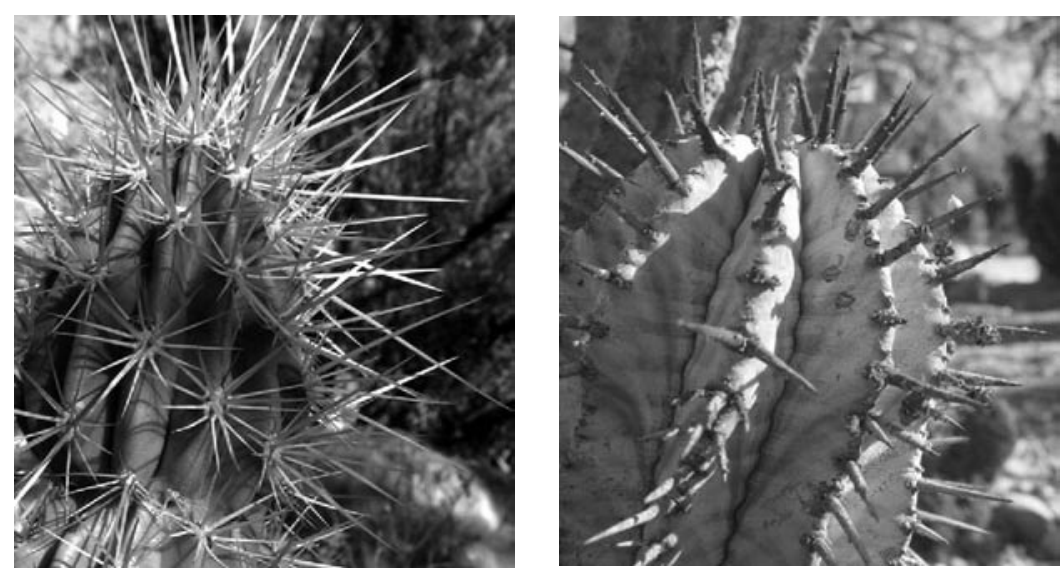

Figure 1. An example of convergent biological evolution. Left: a species of cactus, Echinocereus nicholii, Sonoran Desert, U.S. and Mexico. Right:a species of succulent, Euphorbia horrida, South Africa. (Photos:Tyler Volk.)

Biological convergence is widespread. As detailed in a comprehensive survey by paleontologist Simon Conway Morris (2003), convergence encompasses hundreds of examples, which extend from the minutia of biochemistry up to animal behavior and even intelligence. There have been evolutionary convergences in pigments used for the absorption of light; in camouflage coloration; in queen-centered social structures, such as in bees, ants, and naked mole rats; and in high-level cognition, as in the combinatorial properties of bird song and human music. Even life cycles, such as those of organisms that catastrophically senesce following a round of mating (mayflies, Pacific salmon), exhibit instances of convergence (Volk, 2002). Biological evolution is a pattern-finding process, on various scales, both spatial and temporal, and the patterns that have been evolved can be similar across diverse and independent instances.

In the terminology of Daniel Dennett (1995), evolution can be generalized as algorithmic. The algorithm (or, more poetically, in our terms, recipe) for evolution can be conceptualized as consisting of three main processes: replication, variation, and selection. As these are iterated and stirred, they produce functioning patterns, all the way from social structures down to organisms and cells, and even subcellular molecular machinery. We here 
discuss each process in turn, first for biological evolution, and then move to the analogous processes in cultural evolution.

In the process called replication, new organisms are created that are similar to their ancestors. Replication varies from the mitotic division of single cell creatures, with an exact copying of the genome, to the more complex procedure of sexual reproduction, with a new and unique genome that nonetheless carries much of the same pattern, which is why those of us reading this paper are humans and not chimpanzees. However accomplished, replication is the pattern transfer process of evolution through generations of parents and progeny.

But this pattern transfer, even in asexual reproduction, carries a degree of variation because of mutations. The types of mutations include (among others still being elucidated): simple base pair substitutions of DNA (which occur during about every billion base pair copying events), horizontal gene transfers across species in bacteria and other prokaryotes, position shifts of genome segments within the genome, and whole gene and even whole chromosome duplications. Thus the process of variation creates new forms during some replications, which are "unintended" patterns that are then tested by the third process: selection, usually called natural selection in the realm of biology. Out there, in the competitive ecosystems of nature, patterns that "work" by virtue of their success in specific lifestyle contexts survive to replicate themselves onward into the future, while patterns that don't work, over enough time, terminate.

When the contexts in which the evolutionary algorithm (or recipe) is run are the same or similar, the successful patterns that are generated can be similar. The result is convergence. The forms of American cacti and African succulents have already been noted. As another example, mammalian dolphins and tuna fish are both streamlined not because these creatures derived from a common ancestor that possessed that shape but because evolution honed similar functional forms for lifestyles that require almost constant propulsion in water, in which low-friction, efficient propulsion is rewarded by catching food using less energy or escaping predators quickly.

We can apply the same reasoning to culture. Many scholars have recognized that the generation of functional forms in culture is a process similar to biological evolution in its basic recipe of replication, variation, and selection (Boyd and Richardson, 1985; Bickhard and Campbell, 2003; Mesoudi et al., 2006). The existence of two great evolutionary processes on Earth, one very ancient (biological) and the other much more recent (cultural) and "on top" of the more ancient one, has been emphasized by Blackmore (1999), Bateson (1979), and Wilson (1998), as well as others. Though the dynamics of how cultural evolution operates in detail is still a matter of great interest and active investigation (see, for example, Mesoudi et al., 2006, which includes 
many responses to the target paper), what we need here to continue our logic is merely the fact that the three processes of the general evolutionary algorithm are in play.

There are many ways to replicate cultural patterns: orally through language, kinesthetically through ritual, mechanically through manufacturing from ancient stone tools to modern jets, socially through apprenticeships and community structures, and in printed words and electronically, to name just a few. In cultural evolution there is not an exact analogy to the genotype and phenotype 'nuts and bolts' of biological replication (Bickhard and Campbell, 2003, Blackmore, 1999), but there is indubitably the process of replication. Words, cars, ideas, songs, bicycles, cell phones all get replicated.

Furthermore, like those of biological evolution, the systems created by cultural evolution are subject to variation. Bob Dylan writes and performs his version of "All Along the Watchtower," and then along came Jimi Hendrix who put his own spin on it. Likewise did Neil Young and a dozen others, as can be verified with a search on iTunes and downloaded. Auto designers offer this year's models, which are variations on the previous years', equipped with what they expect are new and improved refinements to the former designs. The same happens to items for sale in the fast food industry, to television reality shows, to books released, and to papers such as this one, which will hopefully be cited and which has been constructed by adding value (again, hopefully) to works we cite. Variation is crucial in the stabilization of social cybernetics, according to Ashby (1956), and our cultural zeitgeist encourages variation in children's art classes, in the rewarding of creativity in the sciences and industries, and in the copyright and patent system.

Selection, the third process in the algorithm of general evolution, has many cultural aspects and angles. There is the self-selection by the visual artist alone in the studio. Then there is selection by editors in book publishing. Employers hire and fire employees, universities admit students or not. For a number of aspects of cultural evolution the ultimate agent of selection is the collective buying public. Consumers in the invisible hand of the marketplace are the analogy to nature's creatures in the ecological struggles for existence, in which the creatures compete both directly in the food chain of predators and prey as well as more indirectly by cooperative symbioses. Turning back to culture, the funds obtained when items are purchased by consumers from the business entities that perform the replications (say, via manufacturing) allow those entities to feed those funds back into furthering the processes of replication and variation. The result is more Starbucks stores, Boeing jets, and Harry Potter books spread throughout the world. Thus culture, from its pottery to its myths and social structures, contains systems, which from populations of slightly different, competing systems, have been selected "to work" in specific contexts of challenge and opportunity. 
Now we come to the relevance of this groundwork for the existence of metapatterns. Systems that are subject to an evolutionary process, whether biological or cultural, often need to perform in a similar way. Specifically, when the systems share similar contexts in which they are being tested, then some aspects of the resulting functioning parts of those systems could be truly analogous. Such shared aspects are metapatterns. The metapatterns are not limited to common patterns only within the giant domains of biology or culture but apply across these domains and even apply to many aspects of cognition, because the mind can be analyzed as evolving both in evolutionary time and within the developmental lifetime of each individual, influenced by the overarching cultural environment (Edelman, 1990; Bickhard and Campbell, 2003).

As we saw in the example in the previous section, columns for support were independently evolved to serve as crab legs, tree trunks, and Eiffel Tower struts. The case of the corn stalk in the Navajo iconography is particularly interesting - and crucial as a case that can be generalized - because a column in biology was taken as a visual and meaning-laden beginning and then replicated in a cultural pattern, in sand painting as it so happens here. The transferred pattern is used as a functional part of a story that involves a path from the underworld to our world. Corn is essential to Navajo wellbeing, both nutritionally and, as the metapattern of a tube for both transfer and support, mythically and psychologically. In this cognitive, metaphoric manifestation, the context of the metapattern no longer includes physical gravity but does involve people's deep need to create shared meaning. Culture has borrowed a form from nature and then elaborated and transformed it into a functional pattern on the cultural plane, which still works as a shape that functions as a 'tube'. This borrowing would not be considered a completely independent invention, but by working (being successful) in two different domains, we could still regard the result of a general pattern that occurs across different domains as a convergence of function. The borrowing is only a means to get patterns into culture, say from nature; that pattern still needs to be tested in the culture and will only thrive if it independently works.

In summary, the concept of convergence is the theoretical underpinning to the existence of metapatterns, the reason we should expect to find them, the foundation that supports our search for them. We have shown why certain functional and structural patterns extend throughout various scales of an inclusive super-domain that includes, at least, biology and culture. We next turn to some examples.

\section{Borders and more metapatterns}

Numerous creations of a metapattern in both biological and cultural evolution can be illustrated with the metapattern called borders. The border is a 
specialized part of a system that functions both to isolate a system from the environment and to regulate the exchange of matter, energy, and information with the environment, usually by the capability to actively open and close pores. The cities of Renaissance Italy, such as Lucca and Florence, had thick, protective perimeter walls perforated with gates to allow people and goods in and out. This generic border design of barriers-and-pores is similar to that used in the membrane that bound a typical eukaryotic cell, with its overall lipid barrier that is perforated with ion channels and receptors.

Borders have been crucial at major transition points in biological evolution. During the origin of life by 3.5 billion years ago, a necessary initial step included a protective lipid membrane, because all cells have such a border. The jump in complexity from the prokaryotic to eukaryotic cell, at about 2 billion years ago, was marked, in part, by the enclosure of the cell's DNA into a membrane-bound nucleus, and the outer border for many eukaryotes (but not prokaryotes) achieved the ability to bring large particles into the cell (ingestion). Then, within the last half billion years, two more border inventions played a role in the move from the water-tied life of amphibians (for reproduction) to fully terrestrial life in the dry air: the reptilian eggshell and scaly skin. Progressing to birds and mammals, the developments of feathers and hair, respectively, helped to control elevated body temperatures, which paved the way toward more sophisticated intelligence.

In culture, although specific borders rise and fall in prominence, new kinds at all scales tend to go along with the development of social complexity. By at least the Upper Paleolithic, humans used animal skins to augment their own biological skins. In the region of today's Ukraine, during the depths of the last ice age, domes of mammoth bones provided shelter. Later, many civilizations and independent city-states were literally given shape by their walls, which facilitated the development of complex human social subsystems within. Today's national borders have their own "skins" that are constructed more by laws and guns than sticks and bricks. National borders are becoming more porous, in some ways, with open trade agreements, but national security to unlawful entry is becoming an increasing concern. Tight packaging, sometimes with zip-lock pores, seems ever on the rise as consumers demand uncontaminated goods. Thus borders are in flux today. We can freely get in a plane and hop across the globe with greater ease in many cases and yet require buildings with precisely controlled internal temperatures, aided by insulated walls and thermal-pane windows.

The structural and functional aspects of borders are a rich source for investigations that involve the classic techniques of contrast and comparison. Perhaps crucial for the future use of metapatterns in educational research is the fact that we conceive of our psychological selves, as well as the selves of others, as utilizing borders to think with and even as a basis for the 
self-structure. Lakoff and Johnson demonstrated this in their "container" metaphor: falling "in" love, keeping an idea "in" mind. Volk (1995) included examples such as "thin- or thick-skinned," "covering your butt," and "having an open mind." We suggest that if the development of one's mind is a case of "neural Darwinism" (Edelman, 1990), such that forming cognitive patterns that work results in learning, via a process of variation and selective retention (Bickhard and Campbell, 2003), then the formation of mental borders of both isolation and exchange are examples of the general border metapattern, along with cell membranes and national borders.

We first used the example of tubes to lead into the rationale for the existence of metapatterns, and now have briefly surveyed examples of borders in roughly chronological evolution, from nature to culture and then to mind. We present here a list of additional metapatterns in a briefest possible format. Most were more fully described in individual chapters by Volk (1995). Bloom (2005b) contributed a number of others noted below. The list is not meant to be exhaustive or unique. Others might be added. Some should probably be understood as sub-metapatterns of others, and in several cases that is explicitly noted. Our point is not to claim to have discerned the taxonomy, but to show that some high level taxonomy is being used by evolved systems. We support one reviewer's comment, that the taxonomy could be used for meaning making. We start with the "spheres" and end with "cycles."

1 Spheres: maximum volume, minimum surface, containment; grapes, domes.

2 Sheets: transfer surface for matter, energy, or information; fish gills, solar collectors.

3 Tubes: surface transfer, connection, support; leaf veins, highways, chains of command.

4 Webs or Networks: parts in relationships within systems (can be centered or clustered, using clonons or holons, see 8, 11, and 12); subsystems of cells, organisms, ecosystems, machines, society.

5 Borders: protection, openings for controlled exchange; cell membranes, national borders.

6 Binaries: minimal and thus efficient system; two sexes, two-party politics, bifurcating decision process.

7 Gradients: continuum of variation between binary poles; chemical waves in cell development, human quantitative and qualitative values.

8 Centers: key components of system stability; DNA, social insect centers, political constitutions and government.

9 Layers or Holarchy: levels of webs, in which successive systems are parts of larger systems; biological nesting from biomolecules to ecosystems, human social nesting, engineering designs, computer software.

10 Emergence: general phenomenon when a new type of functionality derives from binaries or webs; life from molecules, cognition from neurons. 
11 Holons versus clonons: parts of systems as functionally unique versus interchangeable; heart-lungs-liver (holons) of body versus skin cells (clonons) of the skin.

12 Clusters: subset of webs, distributed systems of parts with mutual attractions; bird flocks, ungulate herds, children playing, egalitarian social groups.

13 Arrows: stability or gradient-like change over time; biological homeostasis, growth, self-maintaining social structures.

14 Breaks: relatively sudden changes in system behavior; cell division, insect metamorphosis, coming-of-age ceremonies, political elections.

15 Triggers: initiating agents of breaks, both internal and external; sperm entering egg, precipitating events of war.

16 Cycles: recurrent patterns in systems over time; protein degradation and synthesis, life cycles, power cycles of electricity generating plants, feedback cycles, educational grade levels (cyclic design within an arrow of overall educational progress.

In addition to these brief descriptions, Figure 2 (next page) illustrates the metapatterns via simple iconographic representations. For most of the metapatterns, the icon is not what would be seen, but serves as a device to highlight the essential features of those metapatterns that are relational systems (such as binaries) or occur in time (such as cycles). Many metapatterns are "seen" through our understanding; but we emphasize that they are not only in our understanding. Rather, many of the patterns of our understanding mimic the patterns in nature and in the larger culture, which is efficacious for our survival and wellbeing, or, in many cases, converge to the same functional systems.

This completes our survey of major metapatterns. They usually have several sub-metapatterns (Volk, 1995), and we admit that we are not yet able to unambiguously distinguish a unique set on an uppermost level and the unique sub-patterns of those. The generality of the metapatterns might even make this task impossible. Nonetheless, we suggest that any of these could serve as units in classroom lessons involving metapatterns, and are rich in potential as ways to bridge what is sometimes the isolation of disciplines (thus crossing borders), because they could organize (therefore serve as centers) (a) research questions and data analysis and (b) classroom explorations of form and function. In fact, because examples of the metapatterns are so numerous, students can truly explore them as virtually unbounded territory. And because the metapatterns have psychological manifestations, we would expect to find them playing key roles in the social dynamics of a classroom and in the logical and emotional dynamics of discussions in any educational situation (see Part II). 


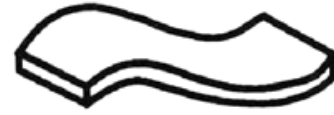

SHEETS

surface transfer

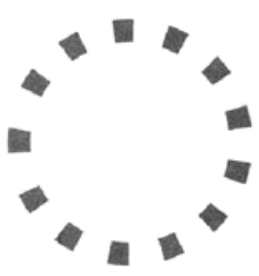

BORDERS

isolation $\&$ exchange

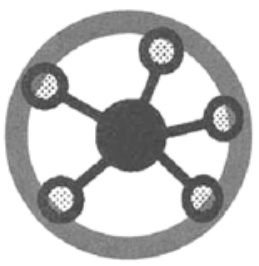

CENTERS

efficient control

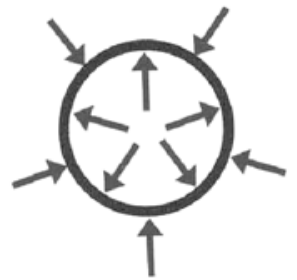

EMERGENCE

the "new" as niche

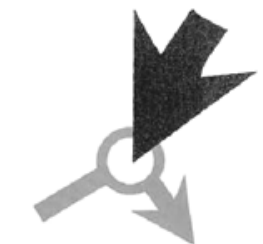

TRIGGERS

amplification

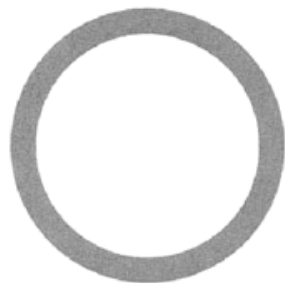

SPHERES

low surface, high volume

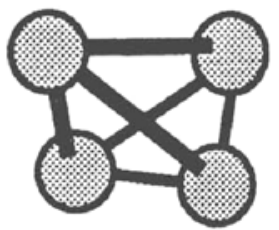

WEBS/NETWORKS

systems of parts and relations

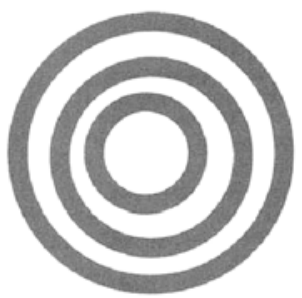

LAYERS multi-scale structural \& functional nesting

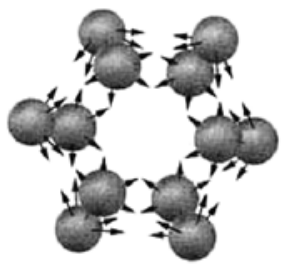

CLUSTERS sub-set of webs, emergence from attraction

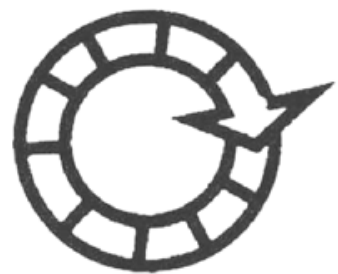

ROWS control

of time, stability, development

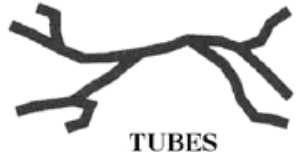

transport, relationship
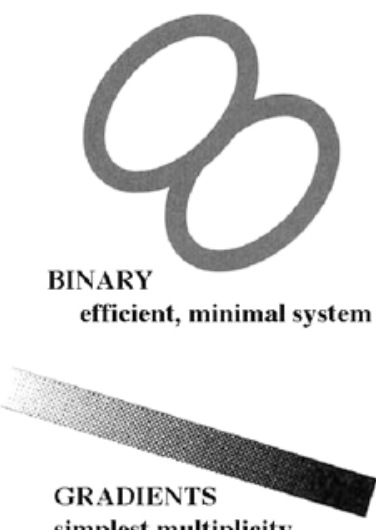

simplest multiplicity

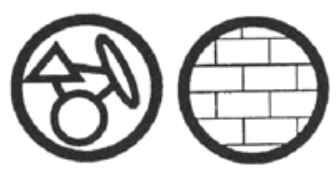

HOLONS \& CLONONS system-building strategies

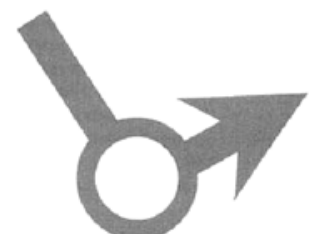

BREAKS

behavioral jump

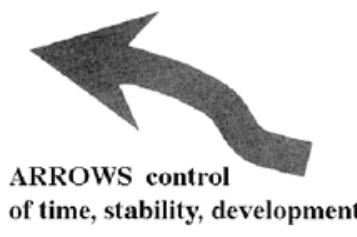

control of time CYCLES via recurrance

Figure 2.The sixteen metapatterns described in the text. 


\section{Metapatterns and patterns from the complexity sciences}

Our working assumption, admittedly not yet rigorously tested, is that metapatterns can serve in the process of learning as templates for understanding systems on a number of different scales, and thus for making connections between these scales. Certainly research into the educational possibilities of metapatterns would be interested, inherently, in a multi-level systems approach.

Another multi-level systems approach, found in numerous articles in this journal and which can be applied to classroom instruction and research initiatives, comes from the complexity sciences, overviews of which are found in Waldrop (1992) and Johnson (2001). Because the principles of the complexity sciences could be seen, in some ways, as competitive alternatives to metapatterns (or visa versa, given the large, established interest in applications from the complexity sciences), we will make a few remarks about the differences in the two approaches and the possibility for synergy.

We here use the overarching term complexity science (or sciences) in the manner of Gilstrap (2005), within which he includes complexity theory, which covers heterogeneous complex adaptive systems, and chaos theory, which describes homogeneous nonlinear, chaotic systems. The complexity sciences, when they were initially created, came from exploring the properties of mathematical simulations of systems with nonlinear interactions and, usually, a large number of similar parts, thus very often in the form of we like to call clonons in the metapatterns language. In the complexity sciences, the systems that emerge from mathematical models of these numerous parts in relationships are general patterns that often cut across phenomena as diverse as molecular dynamics, schooling behavior of birds and fish, fragility of ecosystems, growth of cities, and dynamics of economics, to note just a few. Different mathematically-based sub-disciplines within the complexity sciences include the study of self-organized criticality (Bak, 1996), and the more recently developed science of scale-free, power-law networks, which range from enzymes inside cells to the worldwide web (Barabási, 2003).

Key to the usefulness of the complexity sciences in educational design and research is the fact that these patterns derived from mathematical-physics have often proved useful as metaphors to aid in bettering organization structures, as had been recently overviewed for the classroom environment by Gilstrap (2005). In some cases, insights from nonlinear physics of complex systems, specifically from the work of Prigogine (1997), have even been extended into the epistemology of learning (Osberg and Biesta, 2004).

Many of the key patterns of the complexity sciences are similar to particular metapatterns listed in this paper. For instance, homogeneous systems in chaos theory, such as computer simulations of birds in flocks, 
would be an example of the metapattern that we call clonons in webs or clusters. We have cited emergence as a metapattern, and this term is also a key concept in the complexity sciences (Johnson, 2001; see also Osberg and Biesta (2004) on types of emergence). The heterogeneous systems of complexity theory are similar to the metapattern of holons interacting to form a nested holarchy of functionally distinct parts within wholes. Feedbacks that bring systems, after perturbations, to various kinds of attractors (point, periodic point, periodic, and strange attractors; see Gilstrap, 2005), create the metapattern that we would call the cycle. We also note that to the extent that such attractors create directionality in behavior of systems and then their stabilization, there is an analogy to what we call the metapattern of an arrow, with a goal at the end, its equilibrium state (even if cyclic within that equilibrium).

Continuing, the example of a periodic attractor used by Gilstrap in his annual re-working of a classroom syllabus, which is cyclic but contains annual modifications, can be visualized as a helix, a combination of the metapatterns of arrow and cycle. The bounded stability of a strange attractor in chaos theory has relevance to the metapattern of a border (though not as a specific functional structure, as in many borders), and the famous concept of bifurcation in the complexity sciences, in which a system relatively suddenly shifts from one stable state to another, contains examples of the pair of metapatterns that we call breaks and triggers.

There are clearly a number of overlaps and thus potential synergies between the metapatterns and the patterns of the complexity sciences. Both can aid in the task of what Gilstrap, quoting Morgan (1997, p. 282) says can encourage us "to develop mind-sets and skills that focus on recognizing and changing patterns." We believe that an approach using metapatterns, which keys in to the relationship between form and function, can either precede or follow, and thus complement, the introduction of the general patterns from the complexity sciences in a learning environment, which would have implications for research (see Part II).

But we must emphasize one fairly large difference between the usual discussions that incorporate into educational research the general principles from the complexity sciences and how we see the origin and role of the general principles derived from an approach using metapatterns. Despite the use of the complexity-science derived patterns in qualitative or semi-quantitative analysis of social systems, such as the classroom, those patterns were originally elucidated from the outputs of often very sophisticated and abstract mathematical models. Two of the originators already noted, Prigogine and Bak, for instance, were physicists. In the extension of complexity-science derived patterns to the social sciences, it might be assumed, in some situations in which the math is not available 
or even potentially possible, that the underlying mathematics, were we indeed to know them, are the same as the quantitative models that originally elucidated the patterns. But, alas, often there is no way to check these assumptions.

By contrast, metapatterns can be described in words and images, with the words describing how the forms being thought about are related to certain functions that the systems perform or that the parts within those systems perform. In a sense, the logic of metapatterns is closer to that of Darwin's book The Origin of Species, rather than the mathematical models of physics. The findings from an approach using metapatterns come from observing commonalities in nature, culture, and cognition, and then seeking functional reasons for those convergences. (Such reasons might not be there in any given case of course, because similarities could be fortuitous. This is an issue for the science of metapatterns and as aspect of the critical thinking that would need to be taught to students.)

Metapatterns are derived from observing that scale-crossing patterns are there in the world and have evolved (in the universal sense discussed) because they are clearly functional. In other words, the metapatterns are inherently linked to narratives that describe functions, rather than beginning as mathematical patterns that first were understood from the dynamics of "cells" in numerical models and were then extended into more qualitatively characterized systems such as education.

We wonder what complexity science would have to say, for example, about biological life cycle transitions, such as the transit from tadpole to frog, or from cocoon to butterfly, or during the sexual maturation of organisms, or, moving to cultural evolution, within the various puberty or coming of age ceremonies of different cultures around the world, which are cultural breaks laid on top of a common biological one (Volk, 1995). It seems that the best that a qualitatively extended approach from complexity science could do would be to label these as bifurcations. But these instances are not proven bifurcations in the sense of a system having gone one way or another at a dynamic-mathematical bifurcation point. These "breaks" (using the metapattern term) are life cycle designs that emerged from processes of selection operating on biological life cycles and on the cultural imprinting of social norms, as the case may be, via biological and cultural evolution. With the metapatterns approach, after discerning an interesting and widespread common pattern, one then can start asking functional reasons behind the selection of these patterns: the evolution in insects across graded degrees of metamorphosis, for example. As far as we can tell, there is no language in the complexity sciences to discuss these specific kinds of evolved breaks, because the complexity sciences are not usually wedded to the logic of the general evolutionary algorithm, which, as we 
have outlined, is necessary to understand the generation of patterns in biology and culture.

But now turning the tables, the patterns from the complexity sciences, for their part, can aid the functional approach of metapatterns by showing how mathematics can lead to certain very fundamental patterns in the way systems are structured and behave. Perhaps metapatterns and the patterns of complexity theory can most fruitfully be considered as complementing each other with respect to the larger goal of interdiscursivity, using the term of Davis and Phelps (2005), which is to provide students with multiple ways of attacking problems that are broad-ranging and multi-faceted. Both approaches are examples of comparative dynamics, in the terminology of Stanley (2006). It is possible that because some of the patterns from the complexity sciences are truly pervasive, they could be considered as particular examples (subsets) of some of the metapatterns. For instance, the bifurcations of complexity science could be a specific kind of sub-metapattern of the more general metapattern of breaks.

Though the patterns from the complexity sciences offer the most immediate and obvious comparison with functional metapatterns, we wish to briefly point out other system approaches that should be investigated further to gain perspective on both metapatterns and on the patterns from complexity science, to aid the entire enterprise of bringing systems thinking to the classroom and into educational research. One is the theory of bodygrounded metaphor in cognitive linguistics (Lakoff and Johnson 1980, Lakoff 1990, Johnson 1990). A second is the system of pattern thinking in creative writing developed by Gabriele Rico (1983/2000). A third is the system of highly conceptual architecture patterns staked out by Christopher Alexander (2003). Space prevents us from discussing here the intriguing overlaps between the findings of these researchers and metapatterns.

\section{Conclusions}

Previous work on metapatterns (Volk, 1995, Bloom, 2005a,b; Bateson, 1979) did not explain why metapatterns should be expected to exist. Here we have focused on convergences: As parts interact and are modified to create larger systems, these systems will be selected for certain functional capabilities by the processes of biological and cultural evolution. The result is domain-spanning metapatterns. This logic grounds the existence of metapatterns and the narrative of explanation that can go into a classroom situation, depending on grade level, that might use a metapatterns approach.

We have discussed the metapatterns of both tubes and borders, showing examples of them from biological and cultural to metaphoric and cognitive, and listed a number of other examples of metapatterns. The list is not a 
closed set but rather provided as a starter to show the range of possibilities. Refining the list might best happen as "complicity" in action (Kieren, 2005), as users work together and experiments are run by the users to see what works and what does not in a teaching or research situation. We submit that the metapatterns are, in the terms of Davis and Phelps (2005), (a) transphenomenal, because they are patterns that facilitate the linking of levels, (b) transdisciplinary, because understanding the details of why a metapattern exists on two levels will require learners to gain comfort with the language of different disciplines, and (c) interdiscursive, because the metapatterns are a functional and evolutionary approach, both distinct from, in some aspects, and complementary to, in other aspects, the approach to generalized patterns from the complexity sciences. We support the overall quest of bringing better pattern-thinking to the classroom, which can likely help in the cognitive tasks of classifying, question asking, self-directed inquiring, expanding interest, generalizing across phenomena, and providing a sense of a truly unified universe. In a companion paper (Part II), we show how opportunities arise for using metapatterns to ask new kinds of questions and for forging new analytical frameworks in educational research.

\section{Acknowledgements}

We thank our reviewers for their helpful comments, which prompted us to substantially re-work parts of this paper. We regret that space prevented us from incorporating all of their suggestions for additional citations and discussion.

\section{References}

Alexander, C. 2003. The phenomenon of life: The nature of order, book 1. Berkeley: Center for Environmental Structure.

Ashby, W. R. 1956. Introduction to cybernetics. London: Wiley.

Bahti, M. 2000. A guide to Navajo sandpaintings. Tucson: Rio Nuevo.

Bak, P. 1996. How nature works: The science of self-organized criticality. New York: Copernicus.

Barabási, A-L. 2003. Linked. Cambridge, MA: Plume.

Bateson, G. 1979. Mind and nature: A necessary Unity. New York: Dutton.

Bickhard, M. and D. T. Campbell. 2003. Variations in variation and selection: The ubiquity of the variation-and-selective-retention ratchet in emergent organizational complexity. Foundations of Science. 8:215-282.

Blackmore, S. 1999. The meme machine. Oxford: Oxford University Press.

Bloom, J. W. 2005a. The application of chaos, complexity, and emergent (meta)patterns to research in teacher education. Proceedings of the 2004 Complexity Science and Educational Research Conference (pp. 155-191), Sep 30-Oct 3 - Chaffey's Locks, Canada (http: / / www.complexityandeducation.ca). 
Bloom, J. W. 2005b. Metapatterns - An Overview. Available online at: $\underline{\text { http:/ / elsci. }}$ coe.nau.edu/readarticle.php?article id=22.

Bloom, J. W. 1995. Assessing and extending the scope of children's contexts of meaning: Context maps as a methodological perspective. International Journal of Science Education. 17(2):167-187.

Boyd, R. and P. Richardson. 1985. Culture and evolutionary process. Chicago: University of Chicago Press.

Campbell, J. 1974. The mythic image. New Jersey: Princeton University Press.

Davis, B. and R. Phelps. 2005. Exploring the common spaces of education and complexity: transphenomenality, transdisciplinarity, and interdiscursivity. Complicity: An International Journal of Complexity and Education. 2(1):1-4.

Dennett, D. 1995. Darwin's dangerous idea: Evolution and the Meanings of Life. New York: Simon \& Schuster

Edelman, G. 1990. The remembered present: A biological theory of consciousness. New York: Basic Books.

Eliade, M. 1972. Shamanism: Archaic techniques of ecstasy. New Jersey: Princeton University Press.

Gilstrap, D. L. 2005. Strange attractors and human interaction: Leading complex organizations through the use of metaphors. Complicity: An International Journal of Complexity and Education. 2(1): 55-69.

Johnson, M. 1990. The Body in the Mind: The Bodily Basis of Meaning, Imagination, and Reason. Chicago: University Of Chicago Press.

Johnson, S. 2001. Emergence: The connected lives of ants, brains, cities, and software. New York: Scribner.

Kieren, T. 2005. A perspective on the idea of 'complicity'. Complicity: An International Journal of Complexity and Education. 2(1): 73-76.

Lakoff, G. 1990. Women, fire, and dangerous things. Chicago: University of Chicago Press.

Lakoff, G. and M. Johnson. 1980. Metaphors we live by. Chicago: University of Chicago Press.

Mesoudi, A., A. Whiten and K. N. Lanland. 2006. Towards a unified science of cultural evolution. Behavioral and Brain Sciences. 29(4): 329-383.

Morgan, G. 1997. Images of organization (second ed.) Thousand Oaks, CA: Sage Publications.

Morris, S. C. 2003. Life's solution: Inevitable humans in a lonely universe. Cambridge, U.K.: Cambridge University Press.

Novak, J. D. and D. B. Gowin. 1984. Learning how to learn. New York: Cambridge University Press.

Osberg, D. and G. J. J. Biesta. 2004. Complexity, knowledge and the incalculable: Epistemological and pedagogical implications of 'strong emergence.' Proceedings of the 2004 Complexity Science and Educational Research Conference. 207-227.

Prigogine, I. 1997. The end of certainty: Time, chaos, and the new laws of nature. London: The Free Press.

Rico, G. L. 1976. Metaphor and Knowing. Unpublished doctoral dissertation, Stanford University.

Rico, G. L. 1983/2000. Writing the natural way (orig. ed./rev. ed.). New York: Tarcher/ 


\section{Putnam.}

Stanley, D. 2006. Comparative dynamics: Healthy collectivities and the pattern which connects. Complicity: An International Journal of Complexity and Education. 3(1): 73-84.

Volk, T. 2002. What is death? A scientist looks at the cycle of life. New York: Wiley.

Volk, T. 1995. Metapatterns across space, time, and mind. New York: Columbia University Press.

Waldrop, M. 1992. Complexity: The emerging science at the edge of order and chaos. New York: Simon \& Schuster.

Wilson, E. O. 1998. Consilience: The unity of knowledge. New York: Knopf.

\section{About the Authors}

Tyler Volk is associate professor of biology and science director of the environmental studies program at New York University. Address: Biology Department, 1009 Silver Center, 100 Washington Square East, New York University, New York, NY, 100036688. He has a long-standing interest in the structures and functions of systems at all levels, and, in addition to the book Metapatterns Across Space, Time, and Mind is the author of Gaia's Body: Towards a Physiology of Earth and What is Death?: A Scientist Looks at the Cycles of Life. Phone: 212-998-3736. Email: tyler.volk@nyu.edu. Website: http: / / www.nyu.edu/fas/biology / faculty/volk/index.html

Jeffrey W. Bloom is professor in the Department of Teaching and Learning, College of Education, Northern Arizona University, Flagstaff, AZ 86011. He specializes in elementary science education. His research interests involve complexity in thinking and learning, teaching for complex learning, and classroom and school communities. He can be reached at 928-523-0665 or at jeff.bloom@nau.edu. His personal website is at: http://jan.ucc.nau.edu/ jwb2. His project website is the Exploring Science Site at: http: // elsci.coe.nau.edu.

(C) Copyright 2007. The authors, TYLER VOLK and JEFFREY W. BLOOM, assign to the University of Alberta and other educational and non-profit institutions a non-exclusive license to use this document for personal use and in courses of instruction provided that the article is used in full and this copyright statement is reproduced. The authors also grant a non-exclusive license to the University of Alberta to publish this document in full on the World Wide Web, and for the document to be published on mirrors on the World Wide Web. Any other usage is prohibited without the express permission of the authors. 
